Hydrol. Earth Syst. Sci. Discuss.,

https://doi.org/10.5194/hess-2018-452-AC2, 2019

(c) Author(s) 2019. This work is distributed under

the Creative Commons Attribution 4.0 License.

Interactive comment on "Reconstructing the Salgar 2015 Flash Flood Using Radar Retrievals and a Conceptual Modeling Framework: A Basis for a Better Flood Generating Mechanisms Discrimination” by Nicolás Velásquez et al.

Nicolás Velásquez et al.

nicolas.velasquezgiron@gmail.com

Received and published: 12 January 2019

article [utf8]inputenc [top $=1 \mathrm{in}$, bottom $=1.25 \mathrm{in}$, left=1.25in, right=1.25in]geometry

natbib graphicx xcolor tabularx graphicx adjustbox 


\section{Response to Reviewer 2}

January 12,2019

Manuscript title: Reconstructing the Salgar 2015 Flash Flood Using Radar Retrievals and a Conceptual Modeling Framework: A Basis for a Better Flood Generating Mechanisms Discrimination

Authors: Nicolás Velásquez, Carlos D. Hoyos, Jaime I. Vélez, and Esneider Zapata

We sincerely thank the anonymous reviewer \#1 and Dr. Eric Gaume for their careful and thoughtful reviews. We have taken their considerations into account and have responded to their concerns both in the paragraphs below and within the manuscript. We feel the current manuscript is indeed better thanks to the reviewer comments. Below, the reviewer comments are in black and our comments are in "blue". 


\section{Anonymous reviewer 2}

After taking into account both reviewer comments, we agree with Dr. Eric Gaume the manuscript needed improvements before final publication. The manuscript indeed focuses on the model reconstruction of the Salgar 2015 flood, focusing on trying to understand the main processes leading to the flash flood generation. The data availability in the region does not allow to conduct an in data analysis study. We decided to include a sensitivity analysis to add robustness to the general results. In the current version of the manuscript, there is a detailed explanation regarding the model set-up, parameter selection, and model calibration, a point raised by both reviewers. As described in the current version of the manuscript, some parameters are indeed from literature, and others from model calibration from other basins in the region with similar properties, and from sensitivity analysis. We also agree hydrological models can not account for the complexity of rainfall-runoff at the small scale; we recognize this fact in the discussion, however, we note that the rainfall data and the simulation results suggest the leading processes triggering the flash flood do not lie in the smallest scales. The current version of the discussion and the conclusions directly state the potential weaknesses of the study.

We fully agree with both reviewers the conclusions derived from modeling results have limitations and those were not acknowledged nor discussed properly in the original manuscript. The current manuscript addresses the potential uncertainty of the modeling results in the discussion section, including a comparison with existing literature. Due to the data scarcity in the region of there is an inherent lack of validation of the model that is not possible to resolve directly. To address this issue we included a sensitivity analysis showing the main conclusions regarding process understanding are the same for different model calibrations using parameter values within physically plausible ranges.

Several changes were made to the manuscript to improve the description of the 


\section{Detailed comments}

P1L15 the virtual tracer experiment separates the simulated "runoff" and "subsurface flow" contributions in the model (i.e. fast and delayed contributions), but realworld tracer experiments could provide very different partitioning as illustrated by numerous past geochemical hydrograph separation studies. Simulated processes can not be simply considered as representing effective processes on the considered watershed. This is a much to simplistic point of view on hydrological processes.

We agree with Dr. Gaume regarding the fact that real-world tracer experiments and multiple separation techniques show evidence of a lack of consensus (?). We also know that there are limitations related to the model flow partitioning. Despite those limitations, there exist successful modeling flow partitioning cases (??). Furthermore, we do not intend to represent all the detailed small-scale preferential pathways of water, but rather the first-order approximation of runoff vs. subsurface flow. For this, we use the model results to obtain a conceptual idea about the general processes. We mentioned this explicitly in the conclusions.

P4L103 : difficulting does not exist.

The text was modified following the reviwer comments.

P8L216 I do not know if it is possible to really say that some watershed are geomorphomogically prone to flash floods. At least, several studies (Marchi et al., 2010; Smith et al, 2018) do not show clear relations between geomorphological settings and magnitude of extreme peak. 
We agree that not all the geomorphological properties of a watershed are linked to the occurrence of flash floods, and geomorphology itself does not imply flash floods. But literature and experience suggest that some properties do play a role in the triggering of flash floods (??). In several flash-floods cases in mountainous regions, the slope and the area are mentioned as important (???). Different authors suggest that the slope of the channels (?) influence the travel speed and the transport capacity of channels. Also, the slope of the hills is related to the sediment and debris production during the event (???). We have added some of these references to the comment in the manuscript.

P10L254 : the selected velocities are relatively high, especially for average cross-sectional velocities (see Lumbroso et al., 2012). The provided estimates may be a little high. Are their some films that could help reduce the estimation uncertainties and provide some ideas of possible peak velocities. According to figure 15, the flood extent has been mapped, and probably flood marks identified, along a large part of the main stream. Since the second event has been produced mainly in the upstream part of the watershed, it would have been interesting to base the analysis on some other peak discharge estimates along the main stream and its tributaries. The ability of the proposed model to reproduce the spatial distribution of the flood peaks on the watershed could have then been tested.

About speeds: In local gauged basins with similar characteristics we have recorded peak flow speeds oscillating between 5 and $7 \mathrm{~m} / \mathrm{s}$ during cases still not considered as flash floods (Figure 1). We have included this comment in the manuscript. By assuming an area of $37 \mathrm{~m}^{2}$ and the described peak speeds, we estimate that the observed flash flood peak flow may vary between 185 and 222 $m^{3} s^{-1}$.

About peak discharge in the main stream and tributaries: The hydraulic model works along with the hydrologic model. Because of this, we do have results for all the tributaries and for both events. In the tributaries, the model shows 


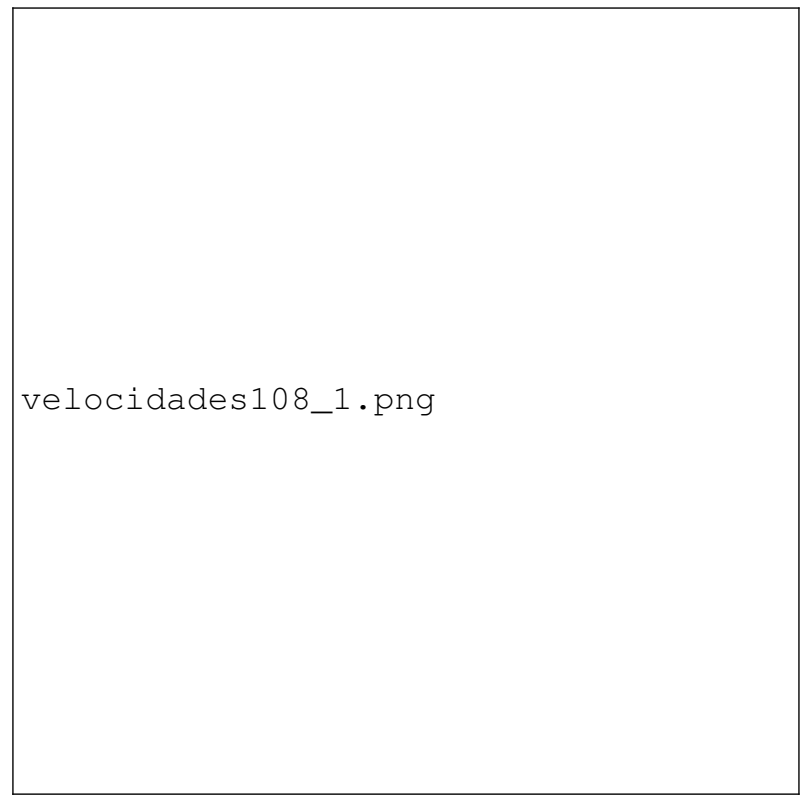

HESSD

Interactive

comment

Fig. 1. Speeds recorded at the Santa Rita Basin located in the Aburra Valley. This watershed has an area of $48 \mathrm{~km}^{2}$ and a mean slope of $45 \%$.

almost no flooding, as observed. And during event 1 the model shows almost no flooded cells.

About the possible test for the model: The main purpose of the test-case in the manuscript was to give a first idea of a low-cost hydraulic-hydrological model and its capability to work in real time with limited data. We do not have other cases in the region to test the model. Nevertheless, the maximum flooded area coincide with the peak flow, and both of them coincide with the reported hour of the disaster.

Printer-friendly version

Discussion paper 
P10L270 the same paragraph is repeated twice. Figure $5:$ impossible to read. The legends must be increased. The five compartments described in the text must be clearly identified.

Figure 5 has been modified and corrected following suggestions from both reviewers.

P13-17 this part could be placed for most of it in an appendix. Moreover, all the variables used in each equation must be clearly defined, which is not always the case and makes it difficult to follow the explanations. The variable names are changing from one equation to the other as for instance $A, A 2, A 3$ between eq. 13-17. If it is the same variable, use the same symbol.

We decided to leave the mentioned text as part of the main document, but we have restructured the section and subsections, and we have made the corrections following suggestions from both reviewers.

P18 The radar rainfall rates must be quality checked. The area is relatively far from the radar and mountainous, two settings that could introduce uncertainties and errors. Are their some available raingauge measurements on the affected watershed or on nearby areas ? How do the radar-based rainfall estimates compare with corresponding raingauge measurements ? The two considered rainfall events are spatially heterogeneous unlike what is stated further in te manuscript. The upper part of the watershed is almost not affected by the first event. It would be essential to distinguish this upper part in the rest of the analysis since the average simulated soil moisture and and runoff components may hide a significant spatial variability. Conclusions drawn on the importance of the first event for the saturation of the soils could be largely nuanced by a more detailed spatial analysis

About rainfall estimates The radar QPE methodology was developed by Sepulveda and Hoyos (2019) using rainfall gauges and disdrometers within the radar

Printer-friendly version

Discussion paper 
domain. Their results indicate that the rainfall estimation works well within a radius of $120 \mathrm{~km}$ (La Liboriana is located at $90 \mathrm{~km}$ ). Despite the distance between HESSD the radar and the basin, and the mountains between them, there are no blind spots in this region for the radar. Figure 2 shows a comparison between the radar QPE and two rain gauges stations installed 3 days after the event The correlation among the hourly precipitation records is 0.65 .

Interactive

comment

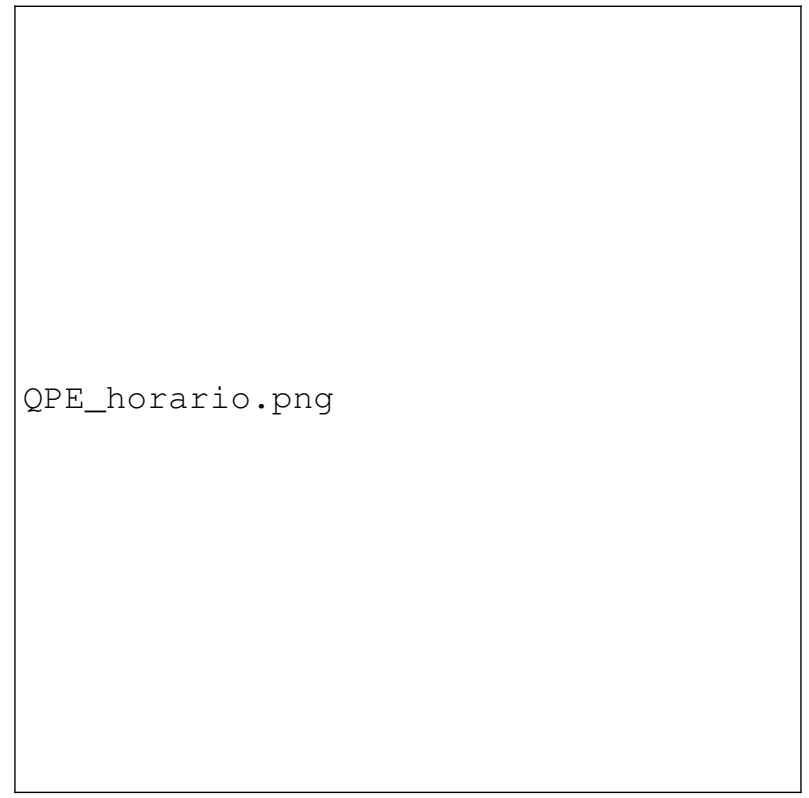

Fig. 2. Hourly rain gauge-radar QPE comparison using two rain gauges installed 3 days after the flash flood event.

About the spatial differences in rainfall We included former Figures 7 and 10 Discussion paper (Figures 5 and 9 in the revised version) in order to asses the spatial variations of 
the variables involved. In these figures, we evaluate the spatiotemporal variations of rainfall and capillary and gravitational storages, and also the amount of saturated and total runoff production. As shown in the figures, there is rain in almost all the basin during Event 1 (about $21 \mathrm{~mm}$ in the upper part of the basin), which increases the overall soil moisture including the upper part of the basin. Due to the rainfall characteristics, there is almost no runoff, which coincides with the fact that there are no reports about the occurrence of flash floods during Event 1.

P18L486 What about the first flood event. Is their any possibility - based on local information - to have an idea of the possible value of the first peak discharge. It would be interesting to know if the simulated discharge $-160 \mathrm{~m}^{3} / \mathrm{s}$ corresponding almost to full-bank flow according to the estimates on page 10, has been observed or no. This would give one more reference point for the evaluation of the implemented rainfall-runoff model.

Unfortunately there is no stage information available from Event 1 . We looked for videos from the community but there is only material available for Event 2. During Event 1 there are no damage reports, but authorities and locals report high fluctuations in the streamflow. This is likely due to the fact that there was no flash flooding during Event 1.

P19L494 It is essential for the second event, according to the spatial rainfall heterogeneities, to provide some distributed simulation results : what part of the flood volume has been produced on the $15 \%$ upstream part of the watershed? Is the contribution form the intermediate watershed significant?

Considering the reviewer comments, we decided to add an additional Figure for Event 2. Figure 3 shows the temporal evolution of discharge during Event 2 in different locations along the watershed's main channel. The upper location corresponds to $15 \%$ of the area of the basin, and the other downstream locations to $52 \%, 76 \%$, and $100 \%$ of the watershed, respectively. In terms of volume, 73 
$\mathrm{Mm}^{3}$ of the total $144 \mathrm{Mm}^{3}$ simulated at the outlet of the basin are generated on the $15 \%$ upstream part of the watershed, corresponding to about half of the total mass. In terms of peak flow, due to the slope and velocity changes, the simulated discharge at the $15 \%$ upstream part of the watershed corresponds to $61 \%$ of the peak discharge at the outlet of the basin.

P21L530 It would be essential to provide some information on the real timing of the floods that could for sure been provided, at least approximately, by eyewitnesses. Discussion on simulated timings, that may be wrong is of little interest.

According to reports from authorities, the peak streamflow reaches the urban perimeter after 2:10 a.m. on May 18th. Some reports state that the peak flow in the most affected community occurred around 2:40 a.m. (https://es.wikipedia. org/wiki/Avalancha_de_Salgar_de_2015). According to our model simulations, the peak flow occurred around 2:20 a.m., which is very accurate considering all the data limitations. The weather radar shows that the rainfall event started at 11:00 p.m. on May 17th.

P21L539 the sentence "Event 1 does not trigger a flash flood event" is not supported by the facts and probably excessive. It did certainly not produce significant overflows and damages, but may have produced a significant flood events (an estimated discharge for this first event is clearly missing in the manuscript). If so, according to the duration of the event, the flood can also be considered as a flash flood.

Given our close contact and interviews with local authorities and the community during the field campaign we conducted to the region immediately after the May 18th flooding, there were no floods reported associated to the event of May 17th.

Part 4 the simulation part, and the interpretation of the results is not uninteresting. But the spatial variability must be shown and commented as suggested before and a clear difference must be made between these simulation results and the realworld. What is presented is the outcome of a numerical model, with the se-

Interactive comment
Printer-friendly version

Discussion paper 
lected parameter and initial state values: some other choices could have provided equally good results if compared to the only available estimated peak discharge but very different flow separation between the various simulated components. Again, the choice of the various parameter values must be clearly justified. Some sensitivity analyses of the results and partitions to these values would also be welcome to strengthened the analyses: are the conclusions always the same if the values are varied over a reasonable range ? I have doubts.

We have restructured some parts of the manuscript, and we have added results from a sensitivity assessment and additional spatio-temporal analysis of Event 2. Taking into account comments made by both reviewers, we added a summary table with the model parameters and an explanation of their origin. Figure 4 shows the results of a sensitivity analysis of the hydrological simulation during the second rainfall event, varying the infiltration rate, and the surface and subsurface speed parameters. The aim of the sensitivity analysis is to evaluate the robustness of the overall results, considering the fact that the quality of some of the watershed information is limited. The overall simulation sensitivity results show the main results described in the previous paragraphs are, in fact, robust to almost all changes in the mentioned parameters, with surface runoff associated with convective rainfall controlling the magnitude of the peak discharge during the Event 2. Changes in the infiltration rate (left panel in Figure 4) result in peak flow changes with a magnitude less than $7 \%$, and changes in the subsurface velocity parameter (right panel in Figure 4) lead to peak flow changes with a magnitude less than $20 \%$ the original simulation. The model highest sensitivity, and hence the largest uncertainty source, appears to be related to the surface speed parameter (middle panel in Figure 4), particularly in the low-end values. Although some of the surface speed values used in the analysis are unrealistically low, it is noteworthy to report that these values lead to the attenuation of the hydrograph and the reduction of the peak flow. 
P22L562 Why should the soils be wet upstream since this area has not strongly been affected by the first rainfall event?

As mentioned before, and as shown in Figure 5 in the revised manuscript, there is rain in almost all the basin during Event 1, about $21 \mathrm{~mm}$ in the upper part of the basin. Also, from Figure 10 in the revised version, we can see variations in the capillary and gravitational storages associated with Event 1 in the higher numbered groups. The capillary storage remains high in almost all the basin until the start of the second event. According to the conceptualization of the model, the gravitational storage and surface runoff start to interact when the capillary storage is full. In this case, this situation is set up by Event 1 . We also made some evaluations for Event 2 using dry initial states, with no flooding in the results (Figure 5).

P24L590 The spatial agreement is not a real surprise since the landslide model has been calibrated and according to the spatial distribution of rainfall.

The reviewer is right in that it might not be a surprise, but it is important to clarify that there was no spatial calibration in order to obtain the right location of the landslides. The calibration only includes the change of the soil depth using a single scalar, constant for the entire basin, in order to maximize the number matching observed and simulated slides. In other words, there is just one single basin-wide parameter modified, and not an independent modification of the parameter for every pixel in order to obtain the right distribution. This is important because in that sense, it serves to check the capability of the model to estimate risk areas only considering topography and rainfall data.

P24L600 Can the concentration of landslides in the first part of the rainfall event be confirmed in any way (eyewitnesses). By the way, this is a surprising result. In general, the landslide density has a general tendency to be related to the rainfall amounts and the progressive increase of soil saturation in mountainous areas

Interactive comment

Printer-friendly version

Discussion paper 
(sign that infiltration dominates the hydrological processes throughout intense storm events). The obtained results would imply that even a very short-lived intense rainfall would have produced landslides in the area. I have many doubts that this is realistic.

Unfortunately, there is no eyewitnesses information available to confirm the landslides in the first part of Event 2. The landslide model determines that a cell is unstable when the perched water table is larger than the critical water table. In the conceptualization of the model, this level is represented by the gravitational storage, which is filled by infiltration. Due to high infiltration rates, our results suggest that the gravitational storage was filled during the start of the rainfall event which eventually triggered the landslides. This type of process is common in the tropics (??).

P25L619 The notions of order are not presented (probably not Strahler order according to the value). Please explain.

The colors of the streams in Figure 16 correspond to the Strahler order of the network. The simulated stream network was delineated by setting a threshold area $\left(0.1 \mathrm{~km}^{2}\right)$ in order to get a match with the observed network in the region.

Figure 15 This figure shows that the post-event survey database is much richer that what is used and presented in the manuscript. Intermediate values of discharges could have been estimated for instance. The comparison between simulated flow depths and observed flood extents is far from perfect. Can this be attributed to the Digital terrain model ? A critical analysis of the digital terrain model could be provided in the manuscript (comparison between observed and extracted cross sections for example)

About the post-event survey: The observed flood spots shown in blue polygons in former Figure 15 correspond to areas delineated from satellite imagery made available by Google days after the flash flood and not to the post-event field trip. 
Most of these affected areas were not accessible after the event so we could not obtained reliable stage information in other locations along the main channel HESSD other than at the outlet of the basin as reported in the manuscript.

About simulated and observed floods: We agree that our results are far from a perfect match with observations. We also agree that a key issue is likely related to the cell size of the available digital elevation model. Nevertheless, our results show a good agreement in different regions of the stream and we consider it a step forward in the development of a low-cost tool for risk management.

P27L647 there is no information about flood 1, the authors can not speak about evidence of remarkable behavioral difference. We do not even know if a flood of significant magnitude occurred... The rainfall can not be described as spatially quasihomogeneous. What do the author mean with "return flow" and "20 groups".

About flood 1: This could be a misunderstanding. As mentioned before (and as it has been made clear in the manuscript), there was only one flash flood event on May 18th. In the manuscript, we talk about two important rainfall events (Events 1 and 2), both leading to the flash flood occurring during event 2.

About the '20 groups' and return flow: As explained in the manuscript, for the spatio-temporal analysis, we divided the watershed cells into 50 groups according to their localization and distance to the outlet. The results of this analysis are shown in Figure 10. In this case, we are talking about the cells near the outlet (the first 20 groups). On the other hand, the return flow is a conceptualization of the model to represent saturation runoff generation. When the gravitational storage is full, the leftover is returned to the runoff.

L654 The authors can not state that the second convective core results mainly in surface runoff. First, the most affected area has hardly been saturated by the first event (spatially detailed result will probably show it) and in anyway, that is a simulation result and not necessarily reality.

Interactive comment

Printer-friendly version

Discussion paper

C14 
See Figures 11 and 12. The spatio-temporal analysis of the simulation reveals a high runoff production on the upper region of the basin. Figure 11 shows that the HESSD convective streamflow accumulation has a behavior similar to the one obtained for the runoff portion of the streamflow. We realize this analysis is obtained from hydrologic model simulations, but it gives us an idea of the processes that might have taken place prior to the flash flood event.

Interactive

comment 


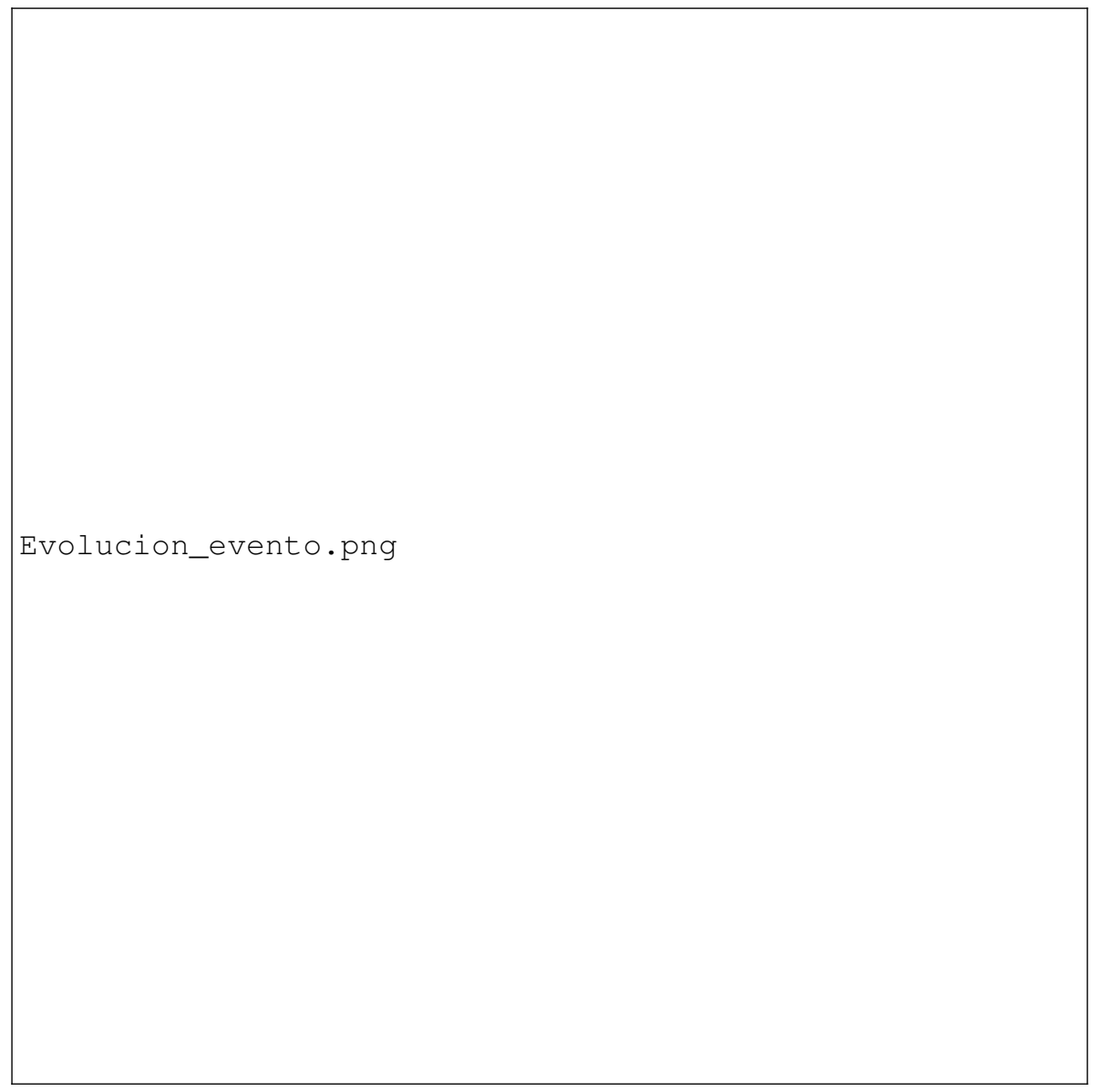

HESSD

Interactive

comment

Printer-friendly version

Fig. 3. Temporal evolution of discharge during Event 2 in different locations along the watershed's main channel. The upper location corresponds to $15 \%$ of the area of the basin, and the other downstream locations to $52 \%, 76 \%$, and $100 \%$ of the watershed, respectively.

Discussion paper

\section{C16}




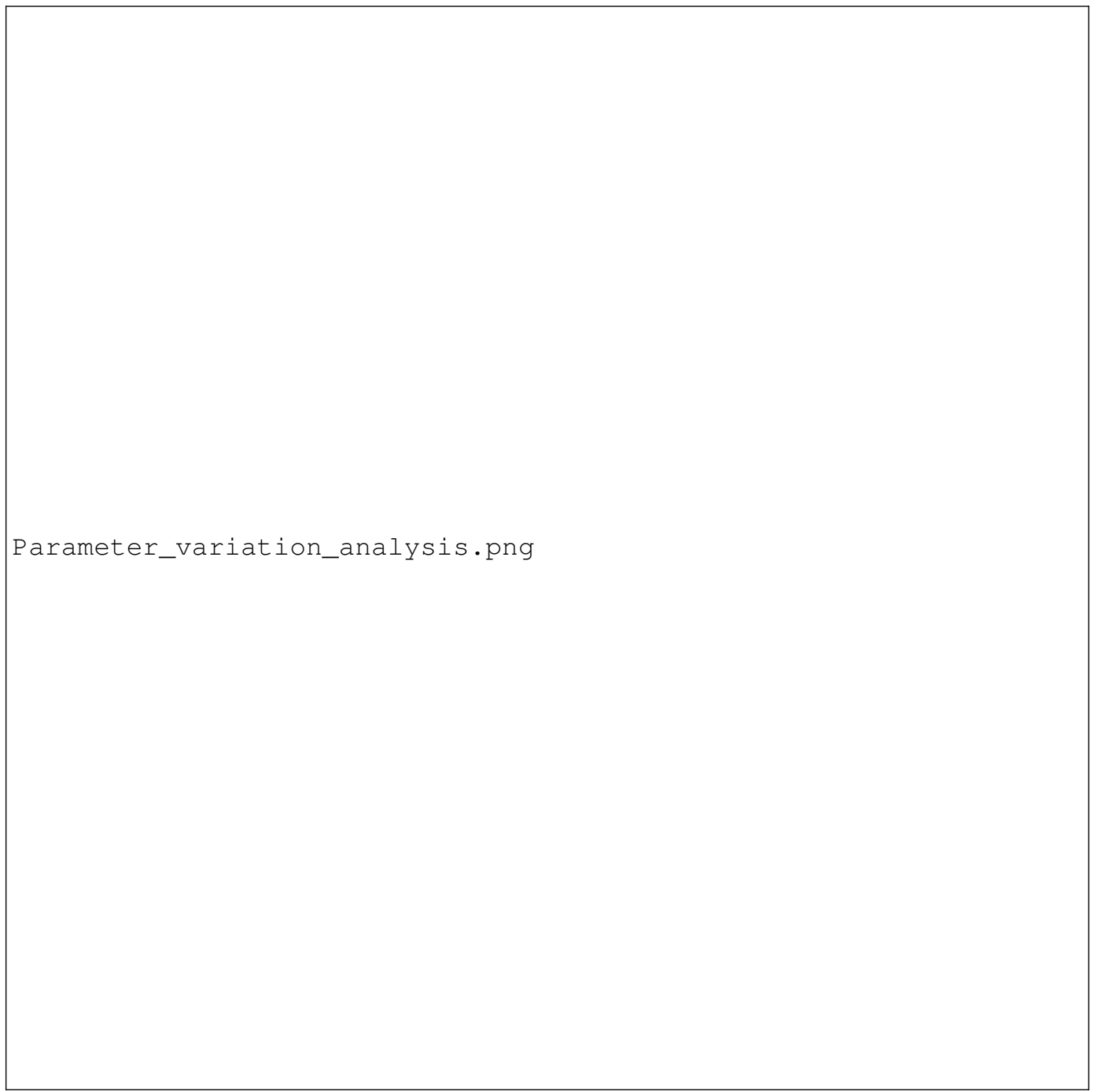

HESSD

Interactive

comment

Printer-friendly version

C17

Fig. 4. Sensitivity analysis of a) infiltration, b) runoff speed and c) subsurface speed parame-

Discussion paper

(c) (1) ters 


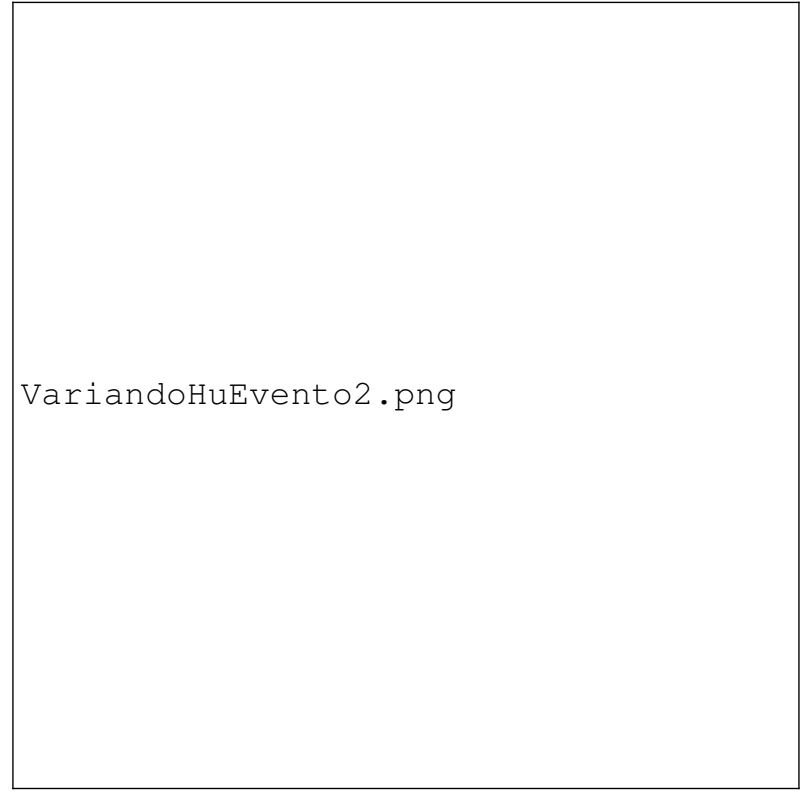

Interactive

comment

Fig. 5. Event 2 simulated varying the percentage of capillary storage in the initial conditions. 